\title{
SÍNDROME DE BROWN-MCLEAN CON LENTE INTRAOCULAR DE CÁMARA ANTERIOR: DESCRIPCIÓN DE UN CASO
}

\section{BROWN-MCLEAN SYNDROME AFTER INSERTION OF AN ANTERIOR CHAMBER INTRAOCULAR LENS: DESCRIPTION OF ONE CASE}

PAREJA-ESTEBAN J ${ }^{1}$, MONTES MA ${ }^{1}$, PÉREZ-RICO C ${ }^{1}$, JIMÉNEZ-PARRAS R ${ }^{1}$, BOLÍVAR G ${ }^{1}$

\section{RESUMEN}

Caso clínico: Se presenta un caso de síndrome de Brown-McLean.

Discusión: Un varón de 71 años de edad desarrolló un síndrome de Brown-McLean tras el implante de una lente de cámara anterior en un ojo afáquico. Se realizó facoemulsificación en el ojo contralateral sin desarrollo de alteraciones en la transparencia corneal. El síndrome de Brown-McLean tiene un origen incierto todavía. Probablemente, se desarrolle en ojos con predisposición genética expuestos a ciertas condiciones, como por ejemplo lentes de cámara anterior.

Palabras clave: Síndrome de Brown-McLean, lentes de cámara anterior, predisposición genética, afaquia, edema corneal.

\begin{abstract}
Case report: We report one case of Brown-McLean syndrome.

Discussion: In this case, a 71-year-old male developed a Brown-McLean syndrome after implantation of an anterior chamber lens in one aphakic eye. A phacoemulsification procedure was performed in the other eye without development of corneal clearance alterations in the next 7 years. The cause of Brown-McLean syndrome is still unexplained. It is possible that it could develop in eyes with a genetic predisposition when these eyes are exposed to certain conditions, such as insertion of an anterior chamber lens (Arch Soc Esp Oftalmol 2007; 82: 315-318).
\end{abstract}

Key words: Brown-McLean syndrome; anterior chamber lens; genetic predisposition; aphakic; corneal edema.

\footnotetext{
Recibido: 31/5/06. Aceptado: 18/4/07.

Servicio de Oftalmología. Hospital Universitario Príncipe de Asturias. Alcalá de Henares. Madrid. España.

${ }^{1}$ Licenciado en Medicina.

Correspondencia:

Jesús Pareja Esteban

C/. José M. ${ }^{a}$ Pereda, n. ${ }^{\circ} 1$, portal $6 .^{\text {a }} 2 .^{\circ} \mathrm{C}$

28806 Alcalá de Henares (Madrid)

España

E-mail: jeparejaes@yahoo.es
} 


\section{INTRODUCCIÓN}

El síndrome de Brown-McLean (SBM) es un cuadro que cursa con edema corneal periférico, respetando la región corneal central, con progresión circunferencial. Sucede generalmente tras extracción intracapsular de cristalino, aunque ha sido descrito tras otras técnicas de cirugía cristaliniana (1) e incluso sin antecedente quirúrgico. Describimos un caso de SBM tras implante secundario de lente intraocular (LIO) de cámara anterior (CA) en paciente afáquico.

\section{CASO CLÍNICO}

Varón de 71 años de edad que consultó para realización de implante secundario de LIO en ojo derecho (OD) y cirugía de catarata en ojo izquierdo (OI). Como antecedente oftalmológico presentaba afaquia de OD tras traumatismo 25 años atrás, el cual motivó la extracción intracapsular de catarata traumática. En la exploración el paciente presentaba una mejor agudeza visual corregida de la unidad, en la escala de Snellen, para el ojo derecho, y de cuenta dedos en ojo izquierdo. Biomicroscópicamente presentaba iridectomía periférica a las 12 horas, vítreo en cámara anterior y afaquia. La transparencia corneal era en los $360^{\circ}$ en ese momento, estando el paciente asintomático. El OI presentaba una catarata NO4-P4-C1. El resto de la exploración oftalmológica era normal. En una primera intervención se realizó facoemulsificación de OI con implante de LIO en cámara posterior bajo anestesia tópica. El postoperatorio cursó sin alteraciones siendo la mejor agudeza visual corregida al mes de la unidad. En un segundo tiempo se realizó vitrectomía anterior con implante de lente de cámara anterior biconvexa de $+18,5$ dioptrías en OD (STORZ, 121UV, PPMA, SN:2YXN17, 6 mm de diámetro), cursando el postoperatorio precoz con normalidad. Dos meses después de la intervención presentaba una mejor agudeza visual corregida de la unidad, encontrándose la LIO de cámara anterior perfectamente centrada. Transcurridos dos años desde la cirugía, el paciente presentó edema corneal periférico con microbullas respetando del eje visual y pigmentación central endotelial marronácea en su ojo derecho (figs. 1 y 2). La paquimetría central era de $520 \mu \mathrm{m}$. El contaje endotelial central tomado con microscopio especular era de 1.246

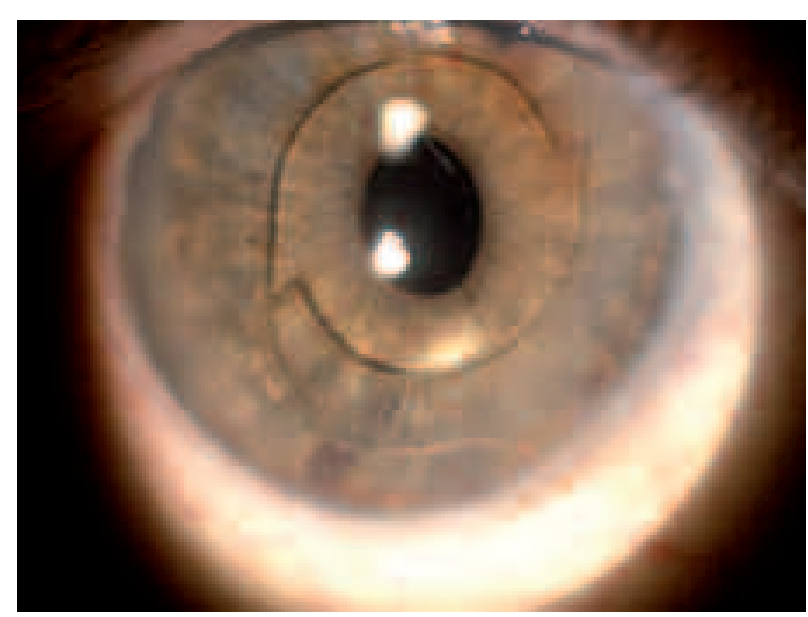

Fig. 1: Edema corneal periférico y LIO de cámara anterior en el SBM.

células $/ \mathrm{mm}^{2}$. Fue instaurado un tratamiento con pomada de cloruro sódico al $5 \%$. Por otro lado el OI no presenta edema corneal, ni periférico ni central, tras siete años desde la intervención.

\section{DISCUSIÓN}

El SBM es una patología poco frecuente descrita a finales de los años sesenta, que cursa con un edema corneal que se extiende por el limbo corneoescleral (2 a $3 \mathrm{~mm}$ ) y que típicamente respeta el eje central de visión. Puede asociarse a una pigmentación endotelial anaranjada o marrón relacionada posiblemente con iridodonesis, ocurriendo de for-

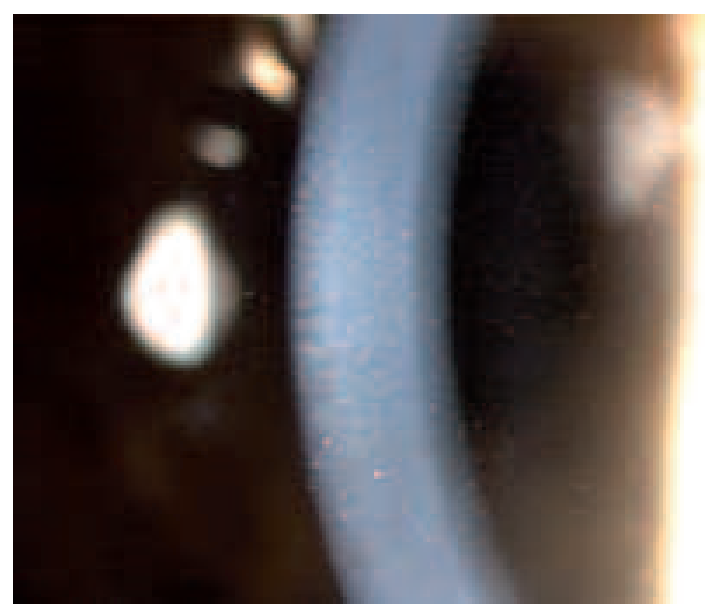

Fig. 2: Pigmentación endotelial marronácea en paciente con SBM. 
ma más frecuente en ojos con LIO en CA (2). Cuando aparecen síntomas suelen ser de sensación de cuerpo extraño y defectos epiteliales.

$\mathrm{Su}$ origen es incierto; se ha visto asociado a diversos tipos de cirugías cristalinianas como la extracción extracapsular de cristalino, facoemulsificación, lensectomía pars plana y vitrectomía, siendo la extracción intracapsular de cristalino la más frecuente (1); si bien ha sido descrito incluso en ausencia de dichas cirugías [reabsorción espontánea del cristalino; subluxación cristaliniana, glaucoma por cierre angular y en un caso de distrofia miotónica (3)]. En nuestro caso destaca el antecedente traumático del ojo derecho que motivó la extracción intracapsular de cristalino.

El tiempo de aparición del cuadro desde el momento de la intervención suele oscilar de 6 a 16 años, habiéndose descrito incluso tras 34 años después (4). En nuestro paciente el tiempo de aparición del edema fue más precoz (2 años) desde el momento del implante de la LIO, aunque el paciente era afáquico desde hacía 25 años.

Han sido descritos otros casos de SBM tras implante de LIO en CA [16\% aproximadamente de los pacientes de la serie de Gothard (1)], en los cuales aunque el edema periférico no coincidía con la situación de los hápticos, al igual que en nuestro caso, estos podrían suponer un agravante para el desarrollo del síndrome (2).

Ha sido descrita del mismo modo la bilateralización del cuadro tras cirugía de ambos ojos, postulándose una predisposición a padecerlo. Tras más de 7 años, el ojo contralateral en nuestro caso mantenía una transparencia total, lo cual conduce de nuevo a la reflexión del agravamiento que supone la presencia de una LIO en CA.

Generalmente, cuando aparecen los síntomas, son tratados con soluciones salinas hipertónicas, lubricación esteroides tópicos habiéndose propuesto las punciones estromales como tratamiento alternativo (5).

En resumen, si bien el SBM es aún de etiología incierta, parece razonable asumir que se trate de un síndrome con una base de susceptibilidad individual (posiblemente genética), en el cuál un factor externo (sirva como ejemplo el implante de LIO en CA), desencadene la clínica.

\section{BIBLIOGRAFÍA}

1. Gothard TW, Hardten DR, Lane SS, Doughman DJ, Krachmer JH, Holland EJ. Clinical findings in BrownMcLean syndrome. Am J Ophthalmol 1993; 115: 729-737.

2. Vote BJ, Grupcheva CN, Ormonde SE, McGhee CN. In vivo confocal microstructural analysis and surgical management of Brown-McLean syndrome associated with spontaneous crystalline lens luxation. J Cataract Refract Surg 2003; 29: 614-618.

3. Rutzen AR, Deen A, Epstein AJ, Maldonado MJ, Hemady RK. Cataract surgery in a patient with Brown-McLean syndrome. J Cataract Refract Surg 2001; 27: 1335-1337.

4. Lim JI, Lam S, Sugar J. Brown-McLean syndrome. Arch Ophthalmol 1991; 109: 22-23.

5. Martins EN, Alvarenga LS, Sousa LB, Orlando Filho VT, Gomes JA, de Freitas D. Anterior stromal puncture in Brown-McLean syndrome. J Cataract Refract Surg 2004; 30: 1575-1577. 